\title{
A new inner tracker based on GEM detectors for the BES III experiment
}

S. Marcello, ${ }^{* a, b}$ M. Alexeev, ${ }^{\mathrm{a}, \mathrm{b}}$ A. Amoroso, ${ }^{\mathrm{a}, \mathrm{b}}$ R. Baldini Ferroli, ${ }^{\mathrm{c}, \mathrm{d}}$ M. Bertani, ${ }^{\mathrm{c}}$ D. Bettoni, ${ }^{\mathrm{e}}$ F. Bianchi, ${ }^{\mathrm{a}, \mathrm{b}}$ A. Calcaterra, ${ }^{\mathrm{c}}$ N. Canale,${ }^{\mathrm{e}}$ M. Capodiferro, ${ }^{\mathrm{c}, \mathrm{f}} \mathrm{V}$. Carassiti, ${ }^{\mathrm{e}}$ S. Cerioni,${ }^{\mathrm{c}} \mathrm{JY}$. Chai, ${ }^{\mathrm{b}, \mathrm{d}, \mathrm{g}} \mathrm{S}$. Chiozzi,${ }^{\mathrm{e}}$ G. Cibinetto, ${ }^{\mathrm{e}}$ F. Cossio, ${ }^{b, g}$ A. Cotta Ramusino, ${ }^{e}$ M. Da Rocha Rolo, ${ }^{b}$ F. De Mori, ${ }^{a, b}$ M. Destefanis,,${ }^{a, b}$ J. Dong, ${ }^{c}$ F. Evangelisti, ${ }^{e}$

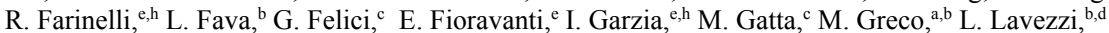
CY. Leng, ${ }^{b, d, g}$ H. Li,${ }^{b, d}$ M. Maggiora, ${ }^{\mathrm{a}, \mathrm{b}}$ R. Malaguti, ${ }^{\mathrm{e}}$ M. Melchiorri, ${ }^{\mathrm{e}}$ G. Mezzadri, ${ }^{\mathrm{e}, \mathrm{h}}$ M. Mignone, ${ }^{\mathrm{b}}$ G. Morello, ${ }^{\mathrm{c}}$

S. Pacetti, ${ }^{\mathrm{i}, \mathrm{l}}$ P. Patteri, ${ }^{\mathrm{c}}$ J. Pellegrino, ${ }^{\mathrm{a}, \mathrm{b}}$ A. Pelosi, ${ }^{\mathrm{c}}$ A. Rivetti, ${ }^{\mathrm{b}}$ M. Savrié, ${ }^{\mathrm{e}, \mathrm{h}}$ M. Scodeggio,,${ }^{\mathrm{e} h}$ E. Soldani, ${ }^{\mathrm{c}}$ S. Sosio, ${ }^{\mathrm{a}, \mathrm{b}}$ S. Spataro, ${ }^{\mathrm{a}, \mathrm{b}}$ E. Tskhadadze, ${ }^{\mathrm{c}, \mathrm{m}}$ S. Verma, ${ }^{\mathrm{e}, \mathrm{h}}$ R. Wheadon ${ }^{\mathrm{b}}$ and L. Yan ${ }^{\mathrm{b}}$

${ }^{a}$ Dipartimento di Fisica, Università di Torino, Via P. Giuria 1, Torino, 10125, Italy

${ }^{b}$ INFN Sezione di Torino, Via P. Giuria 1, Torino, 10125, Italy

${ }^{c}$ INFN Laboratori Nazionali di Frascati, Via E. Fermi 40, Frascati (Roma), 00044, Italy

${ }^{d}$ Institute of High Energy Physics, Chinese Academy of Sciences, 19B Yuquan Lu, Beijing, 100049, P.R.C.

${ }^{e}$ INFN Sezione di Ferrara, Via G. Saragat 1, Ferrara, 44122, Italy

${ }^{f}$ INFN Sezione di Roma, P.le A. Moro 2, Roma, 00185, Italy

${ }^{g}$ Dipartimento di Elettronica e Telecomunicazioni, Politecnico di Torino, C.so Duca degli Abbruzzi 24, Torino, 10129, Italy

${ }^{h}$ Dipartimento di Fisica, Università di Ferrara, Via G. Saragat 1, Ferrara, 44122, Italy

${ }^{i}$ INFN Sezione di Perugia, Via A. Pascoli 14, Perugia, 06123, Italy

'Dipartimento di Fisica e Geologia, Università di Perugia, Via A. Pascoli 14, Perugia, 06123, Italy

${ }^{m}$ Joint Institute for Nuclear Research (JINR), Joliot-Curie 6, Dubna, Moscow Region, 14980, Russia

Published 10 July 2018

A new inner tracker based on a cylindrical gas electron-multiplier detector is under development to replace the current inner drift chamber of the BES III spectrometer. The BES III experiment is carried out at the BEPC II $\mathrm{e}^{+} \mathrm{e}^{-}$collider in Beijing at center-of-mass energies in the tau-charm region with a design luminosity of $1.0 \times 10^{33} \mathrm{~cm}^{-2} \mathrm{~s}^{-1}$. The new inner tracker consists of three cylindrical layers of triple GEM surrounding the interaction point, covering $93 \%$ of solid angle. To fulfill physics requirements, a spatial resolution of $130 \mu \mathrm{m}$ must be achieved. Both planar and

*Corresponding author: simonetta.marcello@unito.it.

This is an Open Access article published by World Scientific Publishing Company. It is distributed under the terms of the Creative Commons Attribution 4.0 (CC-BY) License. Further distribution of this work is permitted, provided the original work is properly cited. 
cylindrical prototypes have been built and tested. A custom ASIC using UMC 110-nm technology has been designed to provide charge and time measurements - the first prototype is in testing. Notable and innovative aspects of the new inner tracker and the performance of the detector prototypes and readout ASIC are reported here.

Keywords: Particle-tracking detectors (gaseous detectors); micropattern gaseous detectors (GEM, MICROMEGAS, etc.); CMOS readout of gaseous detectors; analog and digital electronic circuits.

PACS numbers: 29.40.Gx, 29.40.Cs

\section{Introduction}

The Beijing Electron Spectrometer (BES) III particle-physics experiment ${ }^{1}$ is in progress at the Beijing Electron-Positron Collider (BEPC) II double-ring $\mathrm{e}^{+} \mathrm{e}^{-}$collider at IHEP in Beijing. Operating since 2009, the experiment consists of a wide-ranging physics program spanning a number of topics, including charmonium and open-charm physics, light-hadron spectroscopy, and $\tau$-physics. The program is feasible thanks to the excellent performance of the collider and experimental apparatus. BEPC II operates at a center-ofmass (c.m.) energy between 2 and $4.6 \mathrm{GeV}$, and a design luminosity of $1.0 \times 10^{33} \mathrm{~cm}^{-2} \mathrm{~s}^{-1}$ was recently achieved. The experimental apparatus is a magnetic spectrometer, working with a magnetic field of about $1 \mathrm{~T}$, covering a large solid angle of $\sim 4 \pi \mathrm{sr}$ and composed of different specific detectors. A helium-based main drift chamber (MDC) serves as tracking detector, providing a momentum resolution of $0.5 \%$ for charged particles at $1 \mathrm{GeV} / \mathrm{c}$, a dE/dx resolution better than $6 \%$, and a spatial resolution of $130 \mu \mathrm{m}$ in the $r \phi$ plane. Owing to an increase in luminosity in the last years, the MDC inner layers have worsened in efficiency ${ }^{2}$ by up to $40 \%$, as compared to the value in 2014 .

A promising physics plan to run the experiment until 2022 and beyond has been defined, and a replacement for the inner part of the MDC is thus needed. The detector consists of two parts, inner and outer, which share the same gas volume. The Italian group proposes to replace the eight inner layers with three layers of triple gas electronmultiplier $^{3}$ (GEM) detectors of cylindrical shape.

\section{The BES III Cylindrical GEM Detector}

The cylindrical GEM inner tracker $\left(\right.$ CGEM-IT) ${ }^{4,5}$ for BES III experiment consists of three concentric layers of triple GEMs, as shown in Fig. 1. Each layer contains five regions: the conversion and drift region between the cathode and first GEM foil, with an electric field of $1.5 \mathrm{kV} / \mathrm{cm}$; two transfer regions between the GEM foils, with a field of $3 \mathrm{kV} / \mathrm{cm}$; and an induction region between the last foil and the anode, with a field of $5 \mathrm{kV} / \mathrm{cm}$.

The GEM foil is a metal-coated polymer (50- $\mu \mathrm{m}$ kapton with $3-\mu \mathrm{m}$ copper on the faces), pierced with thousands of holes of $50 / 70 \mu \mathrm{m}$ diameter, with a high field of $10^{5} \mathrm{~V} / \mathrm{cm}$, used for the avalanche multiplication of electrons coming from primary ionization in the drift region. The use of three GEM foils allows a high gain of $10^{3}-10^{4}$ at voltages lower than needed with one or two foils, keeping the discharge probability below a safety value of $10^{-5}$, assuring the electrical stability of the detector. ${ }^{6}$ The 
electrical signal is collected by a segmented anode consisting of two views with $650-\mu \mathrm{m}$ pitch strips, $x$-strips (570 $\mu \mathrm{m}$ wide) parallel to the cylindrical axis, and $v$-strips $(130 \mu \mathrm{m}$ wide) tilted to a large stereo angle greater than $30^{\circ}$, with values varying according to layer position.

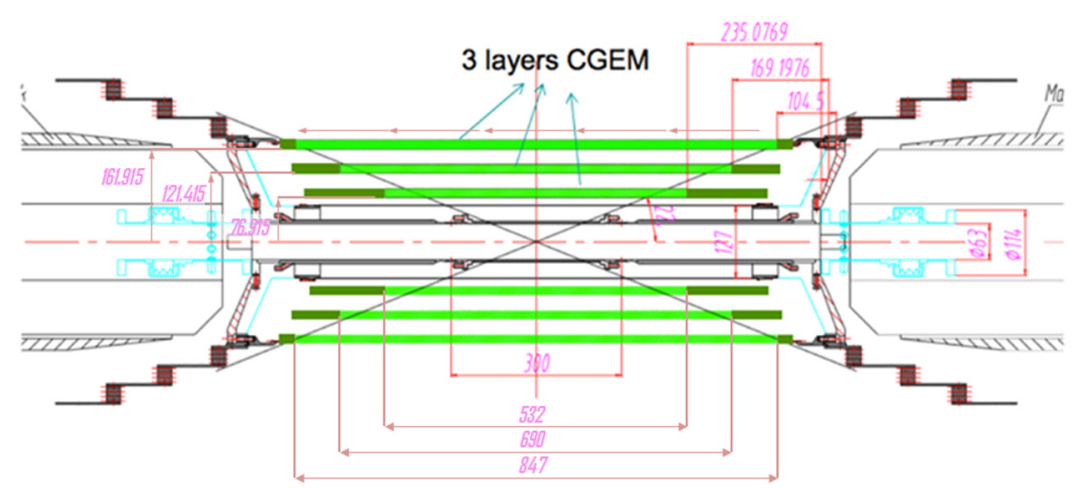

Fig. 1. Scheme of the CGEM-IT (scales are in mm). Courtesy of Ref. 5.

The first cylindrical GEM detector, with four layers of triple GEM and 25000 channels, using the digital readout (single-bit information) of $x v$ strips, was designed and implemented for the KLOE-2 experiment ${ }^{7}$ at the DAФNE $\mathrm{e}^{+} \mathrm{e}^{-}$collider in Frascati. It operates in a magnetic field of $0.5 \mathrm{~T}$, and a spatial resolution of $350 \mu \mathrm{m}$ has been achieved.

In the design of CGEM-IT, three important innovations are introduced:

(i) a lighter mechanical support for cathodes and anodes, based on Rohacell 31 foam instead of honeycomb, to reduce the material budget to a radiation length $\sim 0.33 \%$ of $\mathrm{X}_{0}$ per layer,

(ii) a jagged strip layout of the anode to reduce the capacitance couplings between strips by a factor of $\sim 0.30$,

(iii) an analog readout for which a custom ASIC giving both charge and time measurements was developed. The readout allows loosening of the pitch size, using a reduced number of channels $(\sim 10,000)$. Owing to the high magnetic field of $1 \mathrm{~T}$ in BES III, a digital readout that gives single-bit information on the fired strip is not suitable because it requires high segmentation and a large number of channels.

The CGEM-IT design and analog readout allow keeping the same $r \phi$ spatial resolution of $130 \mu \mathrm{m}$ and the momentum resolution of the current inner tracker, while permitting a better $z$-coordinate resolution $(\sim 1 \mathrm{~mm}$ instead of $2 \mathrm{~mm})$. This advantage improves the vertex resolution of $\mathrm{K}^{0}{ }_{\mathrm{s}}$ and $\Lambda$ particles by a factor of two to three. To proceed with planned experiments, CGEM-IT must be built, fully instrumented, and installed in the BES III spectrometer at IHEP in the summer of 2018. 


\section{Triple-GEM Prototypes}

The GEM signal strongly depends on gas mixture, geometry, and working voltages. For this reason, two kinds of prototypes were prepared to study performance under different conditions. Planar prototypes were tested from 2014 to 2016, followed by a cylindrical prototype of real size at the end of 2016, intended to be used as intermediate layer in the final detector. Both prototypes were studied with pions and muons at the $\mathrm{H} 4$ beam line ${ }^{8}$ of SPS at CERN, where a dipole magnet of up to $1.5 \mathrm{~T}$ with both polarities is available within the RD51 collaboration.

\subsection{The planar triple GEM}

Two planar prototypes of $10 \times 10 \mathrm{~cm}^{2}$ with a 3 - or 5-mm drift gap and an $x y$ segmented anode with a strip pitch of $650 \mu \mathrm{m}$ have been tested. Two gas mixtures have been used: $\mathrm{Ar} / \mathrm{CO}_{2}$ (at a ratio of 70/30) and $\mathrm{Ar} / \mathrm{iC}_{4} \mathrm{H}_{10}$ (90/10). The efficiency plateau with both mixtures reaches about $97 \%$ in both $x$ and $y$ views at a gain of about 6000 , as shown in Fig. 2(a).

Since the new ASIC was not available up to 2016, readout electronics were based on the APV25 hybrid and the scaler readout system, ${ }^{9}$ which can provide the charge and time information for each strip by sampling the charge every $25 \mathrm{~ns}$.

\subsubsection{Study of performance}

The studies reported in Fig. 2 were obtained with the 5-mm drift gap. First, the charge centroid (CC) algorithm, which provides a charge-weighted average of the fired strips in a cluster, was used to measure the position in the hit cluster. A resolution better than $100 \mu \mathrm{m}$ was obtained with both gas mixtures for gain greater than 6000 when the hit strip number is greater than two, as shown in Fig. 2(b).

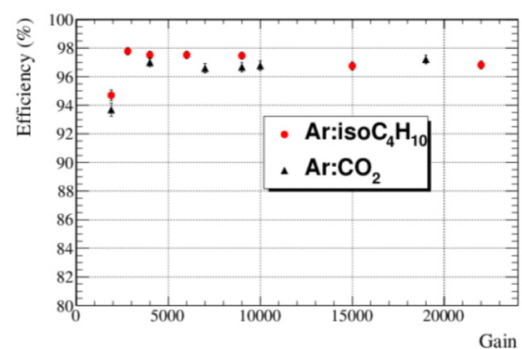

(a)

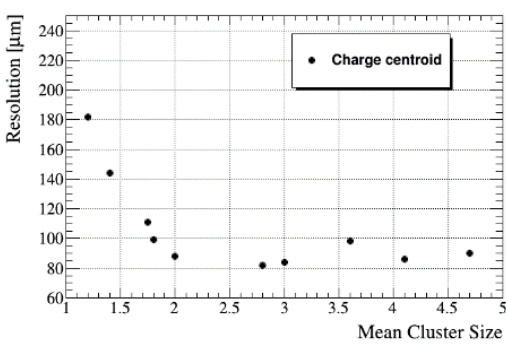

(b)

Fig. 2. (a) Efficiency vs. gain for two gas mixtures, courtesy of Ref. 4. (b) Spatial resolution vs. mean cluster size with $\mathrm{Ar} / \mathrm{CO}_{2}$ gas mixture.

The CC method works properly for orthogonal tracks and magnetic field off when the charge distribution at the anode is Gaussian. For inclined tracks, the cluster size increases, and the charge distribution is no longer Gaussian; therefore resolution using the CC method worsens as a function of the incident angle, as shown in Fig. 3(a). 
The same enlarged cluster size occurs when the magnetic field is switched on, owing to Lorentz force on the electrons, which deteriorates spatial resolution as shown in Fig. 3(b) (keeping orthogonal tracks).

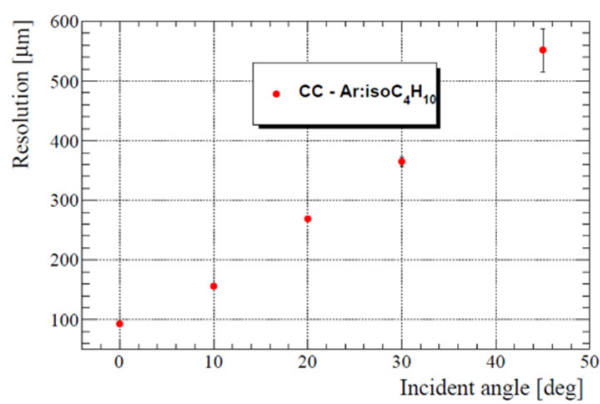

(a)

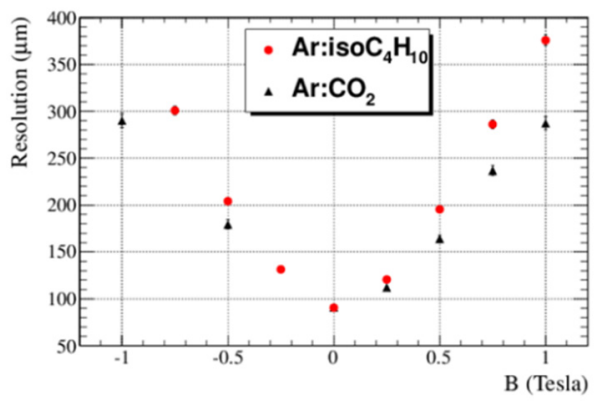

(b)

Fig. 3. Spatial resolution, courtesy of Ref. 5, with CC method (a) vs. incident angle and (b) vs. magnetic field.

To improve resolution, a different method was investigated: micro-TPC ( $\mu$ TPC), which has been successfully applied in a Micromegas detector to upgrade the ATLAS muon system. ${ }^{10}$ Here a drift gap of $5 \mathrm{~mm}$ is seen as a miniature time-projection chamber, and local tracking can be done by exploiting the time information measured on the single strip and the drift velocity from Garfield simulations, as described in Refs. 4, 5, and 10.

The spatial resolutions obtained with the CC and $\mu$ TPC methods, as a function of incident angle (with magnetic field off) and of the magnetic field (keeping orthogonal tracks), are shown in Figs. 4(a) and 4(b), respectively. Clearly, each method succeeds where the other fails in providing good spatial resolution.

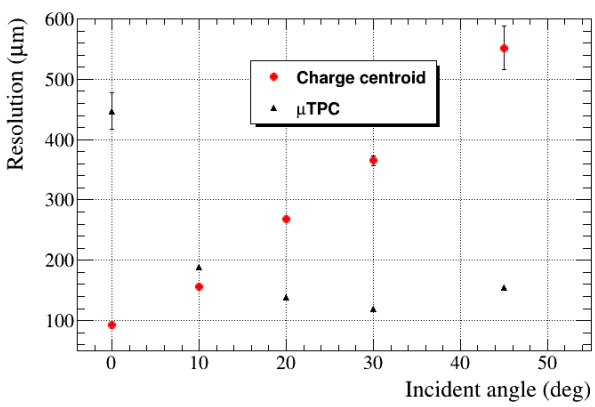

(a)

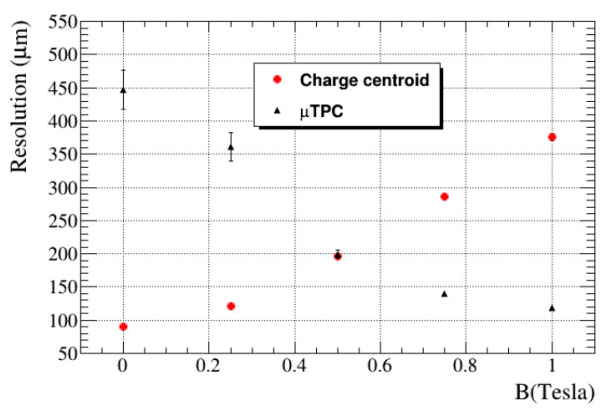

(b)

Fig. 4. Spatial resolutions with CC and $\mu$ TPC methods (a) vs. incident angle, courtesy of Ref. 4, and (b) vs. magnetic field. 
A more complicated and realistic case is to have an incident angle different from zero and the magnetic field on, simultaneously. Here the Lorentz angle between the drift direction of the electrons (under the influence of the magnetic field) and the electric field becomes crucial, and two different effects can be observed. Depending on whether such an angle is concordant or discordant with the incident angle, a focusing or defocusing effect occurs on the cluster size, which shrinks or widens, respectively, as shown in Fig. 5.
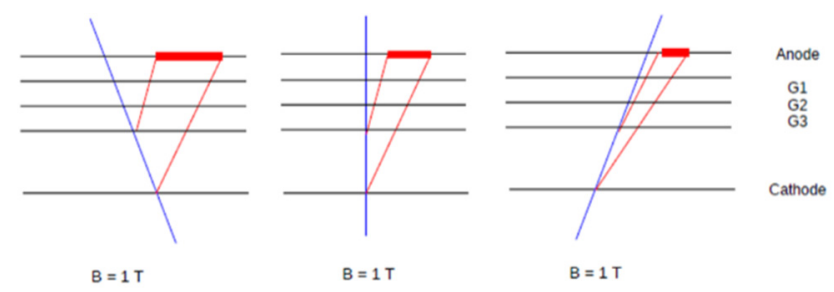

Fig. 5. Defocusing and focusing effects on cluster size (in red) for inclined tracks (in blue), crossing the planar triple GEM. G1, G2, and G3 correspond to the three GEM foils, courtesy of Ref. 5. See text for details.

A measurement of spatial resolution as a function of different incident angles in a magnetic field of $1 \mathrm{~T}$ and with an $\mathrm{Ar} / \mathrm{iC}_{4} \mathrm{H}_{10}$ gas mixture is shown in Fig. 6(a). Both methods, $\mathrm{CC}$ and $\mu \mathrm{TPC}$, were used to reconstruct the position. The range of angles between $15^{\circ}$ and $35^{\circ}$, where the $\mathrm{CC}$ method provides a better performance, corresponds to the focusing effect on cluster size, where the angle is concordant with the Lorentz angle, or about $26^{\circ}$ at $1.5 \mathrm{kV} / \mathrm{cm}$ of drift field with an $\mathrm{Ar} / \mathrm{iC}_{4} \mathrm{H}_{10}$ gas mixture (see dotted line in Fig. 6(a)). Outside this range, the $\mathrm{CC}$ method fails to give good resolution, and $\mu \mathrm{TPC}$ achieves best performance. A combination of the two methods is needed to keep the spatial resolution stable at around $130 \mu \mathrm{m}$ in the full range of incident angles with magnetic field on. This spatial resolution is the best achieved worldwide with triple GEM detectors operating in a high magnetic field of $1 \mathrm{~T}$.

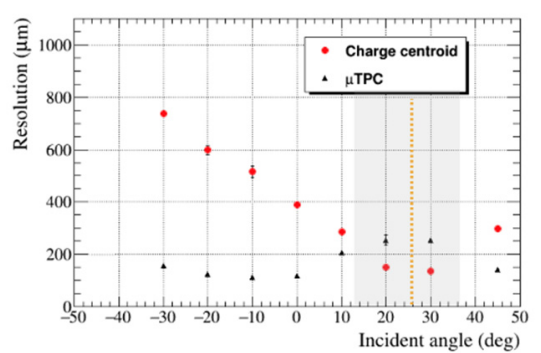

(a)

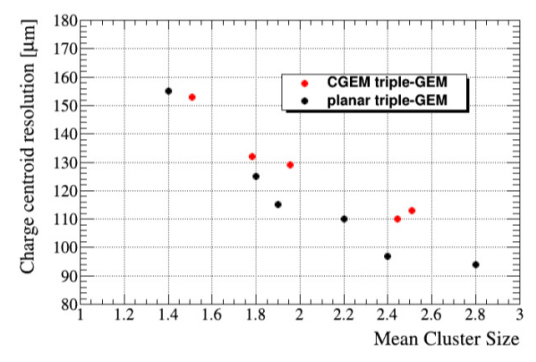

(b)

Fig. 6. (a) Spatial resolution vs. incident angle with a magnetic field of $1 \mathrm{~T}$ for planar triple GEM. (b) Spatial resolution with CC method for planar and cylindrical triple-GEM prototypes with magnetic field off. 


\subsection{The cylindrical triple-GEM prototype}

A cylindrical triple-GEM prototype of real size (that is, with a $12-\mathrm{cm}$ radius and $70-\mathrm{cm}$ length, serving as the intermediate layer of the CGEM-IT detector with a 3-mm drift gap) has been built and tested at $\mathrm{H} 4$ beam line of SPS and operated with an $\mathrm{Ar} / \mathrm{CO}_{2}$ gas mixture with magnetic field on and off.

\subsubsection{Study of performance}

A study of the collected charge and spatial resolution for orthogonal tracks as a function of mean cluster size has been conducted for different values of gain. The $\mathrm{CC}$ algorithm has been used for position reconstruction. The results are compatible with those obtained for a planar triple-GEM prototype with magnetic field off, as shown in Fig. 6(b) above. Of particular note is the achievement of a spatial resolution of $110 \mu \mathrm{m}$. To check electrical stability, the cylindrical GEM was tested under extreme conditions of gain (up to $10^{5}$ ) and beam intensity (with flux up to tens of $\mathrm{kHz} / \mathrm{cm}^{2}$ ) and showed stable behavior without current peaking problems.

Study of the $\mu$ TPC algorithm in combination with the CC method to optimize spatial resolution will be performed as part of extensive testing underway at CERN, using a second cylindrical layer of real size as part of the CGEM-IT's first inner layer.

Meanwhile, for the new layers under construction, a larger drift gap of $5 \mathrm{~mm}$ has been chosen to improve performance with inclined tracks. The $\mathrm{Ar} / \mathrm{iC}_{4} \mathrm{H}_{10}$ gas mixture will also be used to assure higher electrical stability, a higher number of primary electrons, and suitable diffusion features for the optimization of the reconstruction algorithm.

\section{The TIGER ASIC}

Analog readout of the CGEM detector enables the use of charge-centroid and $\mu$ TPC methods to improve resolution up to at least $130 \mu \mathrm{m}$ and allows loosening of the strip pitch to $650 \mu \mathrm{m}$, reducing the total number of channels to about 10,000. To fulfill this purpose, a dedicated front-end ASIC, ${ }^{11}$ providing charge and time measurements and featuring a fully digital output, has been designed, taking into account the specific inputsignal characteristics of the BES III CGEM detector. It should operate with an input dynamic range between 1 and $50 \mathrm{fC}$, a sensor capacitance of up to $100 \mathrm{pF}$ (depending on strip length), a power consumption within $10 \mathrm{~mW} /$ channel, and an event rate of $60 \mathrm{kHz} /$ channel. A time resolution better than $10 \mathrm{~ns}$ is required, and the digital backend of the chip should be radiation tolerant for single-event upset (SEU).

The Torino Integrated GEM Electronics for Readout (TIGER) ASIC is a 64-channel mixed-mode circuit fabricated with UMC CMOS 110-nm technology, with a die area of $5 \times 5 \mathrm{~mm}^{2}$ and a low-voltage operation of $1.4 \mathrm{~V}$. It includes reference and bias generators, internal test pulse-calibration circuitry, and a digital global controller. A functional block diagram of one channel of the chip is shown in Fig. 7. The input stage of the chip consists of a charge-sensitive amplifier coupled to a dual-branch shaper stage. The time-branch 
shaper peaking time is optimized for the time measurement with a leading-edge threshold, while the energy-branch shaper provides a slower signal, enabling better integration and noise optimization for the charge measurement. The very front-end stage is followed by a mixed-mode back end that extracts and digitizes the time stamp and the charge of the input signal. The back-end design is an upgraded version of the TOFPET2 chip, ${ }^{12,13}$ developed for PET in medical applications. This upgrade includes SEU protection using Hamming code and triple modular redundancy.

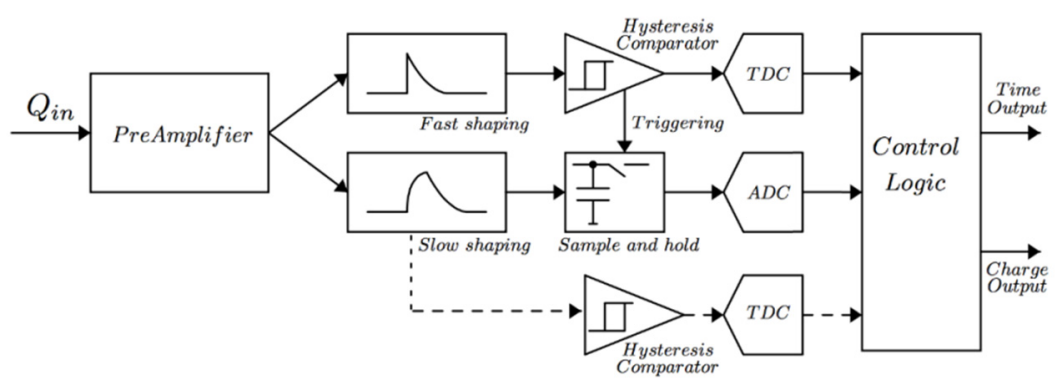

Fig. 7. Block diagram of one channel of TIGER ASIC, courtesy of Ref. 11.

The outputs of the two shapers are fed into two discriminators with different, programmable thresholds, set by a 6-bit DAC with programmable range and LSB, allowing a dual-threshold readout mode. Each channel is equipped with two low-power TDCs, based on interpolators, a sample-and-hold (S\&H) circuit for peak-amplitude detection, and a 10-bit Wilkinson ADC.

The time readout, using both single and double thresholds, provides a time stamp on the rising and falling edges of the signals produced by the fast and slow shapers, respectively, by means of the TDC and 10-bit ADC. The energy readout is performed either by time over threshold or digitization of the voltage amplitude from the S\&H circuit using the same 10-bit ADC as used for the time measurement. Event data is sent to a digital block running at $160 \mathrm{MHz}$, which controls chip operations, handles channel and global-register configurations, and manages off-chip data transmission through two LVDS output links.

\subsection{Performance of TIGER ASIC}

The first tapeout in silicon of the TIGER prototype with a multiproject-wafer shuttle was performed in May 2016, and study of its performance began a few months later. The back-end electronics were found to work properly, as expected. In particular, the S\&H circuit used for charge measurement was tested by injecting a known input charge $\mathrm{Q}_{\text {in }}$ with an external pulse generator and exhibits a linearity better than $0.2 \%$ up to an input charge of $50 \mathrm{fC}$ (see Fig. 8). Concerning time measurement, the quantization error of the TDCs is less than 50 ps rms, which means time resolution may be limited only by the sensor and the very front-end response. 


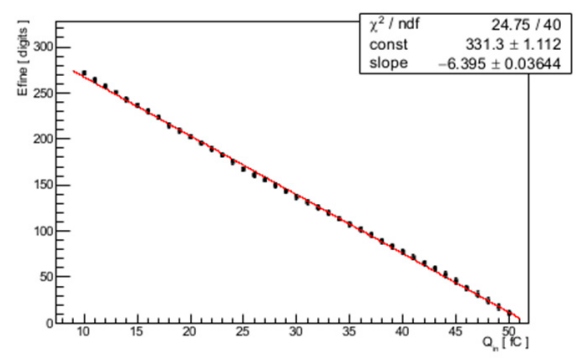

Fig. 8. Charge measurement with the sample-and-hold circuit, courtesy of Ref. 11.

The timing performance of the analog front end was assessed by measuring the jitter of the time branch as a function of different input capacitances. The measured time jitter is below $4 \mathrm{~ns}$ for an input charge of $3 \mathrm{fC}$ with a capacitance of $100 \mathrm{pF}$. A direct measurement of the amplifier gain of one of the channels is shown in Fig. 9(a). The result of $10.4 \mathrm{mV} / \mathrm{fC}$ is in good agreement with the chip's post-layout simulation.

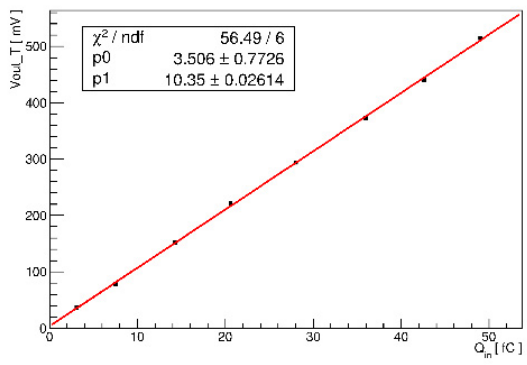

(a)

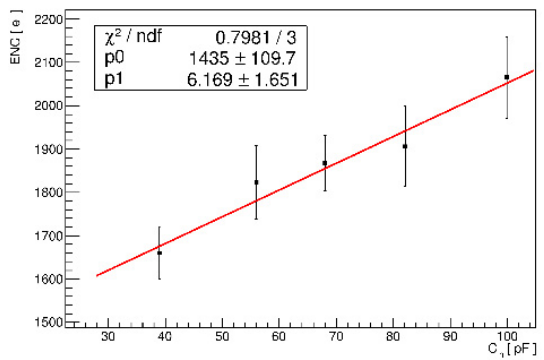

(b)

Fig. 9. (a) Gain measurement of one channel, courtesy of Ref. 11; (b) Noise charge of slow shaper output vs capacitance.

By contrast, in the energy branch, the measurement of the equivalent noise charge (ENC) of the slow shaper output as a function of input capacitance (see Fig. 9(b)) gives a value of about 2100 electrons rms at $100 \mathrm{pF}$-about $50 \%$ greater than that expected from simulations. Comparing the fit of the ENC data, shown in Fig. 9(b), with the fit of simulated data (not shown), similar slopes have been found but with different offsets. Such a difference indicates that the measurement is affected by common mode noise, which is not taken into account in the simulations. Moreover, a baseline dependence on the temperature has been observed, using an analog output available in the ASIC. The baseline level has been found different from the value set through a dedicated pad, and sensitive to the temperature. The root cause has been identified as the fragility of the bias conditions of the holder circuit. An improved design is under study. 
A test of TIGER coupled to the cylindrical GEM prototype with cosmic rays and radioactive ${ }^{90} \mathrm{Sr}$ source was recently begun and is still in progress. Since the performance of the chip is adequate to meet requirements, a second prototype is not needed. The engineering version of the chip is planned for the end of July 2017, with a few minor revisions.

\section{Conclusions}

An innovative cylindrical GEM detector for the BES III experiment is under construction to replace the inner part of the MDC, which has been compromised by aging. To achieve the required spatial resolution of $130 \mu \mathrm{m}$, the analog readout of each strip will be performed by a custom ASIC in UMC 110-nm CMOS technology, featuring a fully digital output.

Exploiting the capability of the ASIC to provide both charge and time measurements, a combination of two complementary algorithms, the charge centroid and $\mu$ TPC, will be used to achieve the required spatial resolution. Several beam tests have been conducted to study the performance of planar and cylindrical triple-GEM prototypes. It is shown that the design resolution of the planar prototype is feasible for inclined tracks and with a high magnetic field of $1 \mathrm{~T}$, using a combination of the two readout methods. Study of the cylindrical-prototype resolution is still underway.

Electrical characterization of the first ASIC prototype shows good performance; only minor revisions are needed for the final engineering run. The whole detector with three layers of triple GEM will be fully instrumented and ready to install in the experimental apparatus at IHEP in summer 2018.

\section{Acknowledgments}

The research leading to these results was performed within the BESIIICGEM project, funded by the European Commission in the call H2020-MSCA-RISE-2014.

\section{References}

1. BESIII Collaboration, Nucl. Instr. Meth. A614, 345 (2010).

2. MY. Dong et al., Chinese Phys. C40, 016001 (2016).

3. F. Sauli, Nucl. Instr. Meth. A386, 531 (1997).

4. R. Farinelli et al., to appear in 2016 IEEE NSS/MIC Conference Record, arXiv:1707.04456.

5. L. Lavezzi et al., J. Instrum. 12, C07038 (2017).

6. S. Bachmann et al., Nucl. Instr. Meth. A479, 294 (2002).

7. A. Balla et al., Nucl. Instr. Meth. A845, 266 (2017).

8. http://sba.web.cern.ch/sba/BeamsAndAreas/resultbeam.asp?beamline=H4.

9. M. J. French et al., Nucl. Instr. Meth. A466, 359 (2001).

10. M. Iodice, J. Instrum. 9, C01017 (2014).

11. M. Da Rocha Rolo et al., J. Instrum. 12, C07017 (2017).

12. M. D. Rolo et al., J. Instrum. 8, C02050 (2013).

13. A. Di Francesco et al., Nucl. Instr. Meth. A824, 194 (2016). 